\title{
Effects of a New Mattress and Pillow and Standard Treatment for Nightly Pelvic Girdle Pain in Pregnant Women: A Randomised Controlled Study
}

\author{
Caroline Feldthusen ${ }^{1,2}$ \\ Monika Fagevik Olsen ${ }^{1,2}$ \\ Hasse Ejnell ${ }^{3}$ \\ Helen Elden (iD) 4,5 \\ 'Region Västra Götaland, Department of \\ Physical Therapy, Sahlgrenska University \\ Hospital, Gothenburg, Sweden; ${ }^{2}$ Institute \\ of Neuroscience and Physiology/ \\ Physiotherapy, Sahlgrenska Academy, \\ University of Gothenburg, Gothenburg, \\ Sweden; ${ }^{3}$ Region Västra Götaland, \\ Department of Rhinolaryngology, Head \\ and Neck, Sahlgrenska University \\ Hospital, Gothenburg, Sweden; ${ }^{4}$ Region \\ Västra Götaland, Department of \\ Obstetrics and Gynaecology, Sahlgrenska \\ University Hospital, Gothenburg, \\ Sweden; ${ }^{5}$ Institute of Health and Care \\ Sciences, Sahlgrenska Academy, \\ University of Gothenburg, Gothenburg, \\ Sweden
}

Correspondence: Helen Elden Diagnosvägen I5, Gothenburg, 416 85, Sweden

Tel +46-702887882

Email helen.elden@vgregion.se
Objective: To evaluate the efficacy of a memory-foam mattress and pillow plus standard treatment for nightly pelvic girdle pain (PGP) during pregnancy.

Method: In this randomised controlled study conducted at a tertiary-care hospital, 66 pregnant women who had nightly PGP were enrolled to receive standard treatment with the mattress and pillow (intervention group, $n=34$ ) or solely standard treatment (control group, $n=32$ ). The primary outcome was change in nightly posterior PGP on a visual analogue scale, VAS from baseline to 4 weeks. Secondary outcomes include nightly anterior PGP, the evening PGP score, estimated sleep duration, number of nightly wake-ups, daytime sleepiness (Epworth sleepiness scale), function (Pelvic Girdle Questionnaire), health-related quality of life, and pain catastrophizing.

Results: Forty-four women (67\%) completed the treatment. The difference in nightly posterior pain intensity was significantly different in favour of the intervention group (VAS, $16.5 \mathrm{~mm}$ $(95 \%$ CI 1.4:31.6) $\mathrm{p}=0.028)$. Sleep duration increased within both groups (intervention group: $26 \mathrm{~min}, \mathrm{p}=0.022$; control group: $14 \mathrm{~min}, \mathrm{p}=0.014$ ) and the difference between groups was significant $(\mathrm{p}=0.046)$. In addition, the intervention group indicated a decreased evening PGP intensity $(p=0.008)$ and fewer nightly wake-ups $(p=0.049)$. The control group showed a deterioration in function (Pelvic Girdle Questionnaire) $(\mathrm{p}=0.018)$ and an increase in daytime sleepiness (Epworth sleepiness scale) $(\mathrm{p}=0.021)$ from baseline to 4 weeks.

Conclusion: In conclusion, significantly lower nightly posterior PGP intensity was noted after the use of a mattress and pillow as an adjunct to standard treatment. Nightly PGP can have adverse effects on various aspects of the health and quality of life of pregnant women, and although the results of this study should be interpreted with caution considering the high drop-out rate and the inadequate statistical power, the findings indicate the potential for the use of such interventions to improve PGP in pregnant women.

Keywords: nightly pain, pregnancy, pelvic girdle pain, randomised controlled trial, sleep disturbance

\section{Plain Language Summary}

Why was the study done

- About $30-40 \%$ of all women who are pregnant worldwide suffer from Pelvic girdle pain (PGP).

- PGP usually debuts during gestational weeks $12-24$ and typically worsens as the pregnancy proceeds. 
- Women with PGP commonly report significant absenteeism from work, difficulties with sexual life, and increased nightly PGP when turning in bed, resulting in frequent awakenings that often lead to daytime tiredness and increased difficulties in performing normal daily activities.

- There are no curative treatments for PGP during pregnancy, and no studies have evaluated treatments for nightly PGP.

What did the researchers do and find

- In this randomised controlled study conducted at a tertiarycare hospital, we evaluated the efficacy of a memory-foam mattress and pillow plus standard treatment for nightly pregnancy-related PGP.

- Nightly posterior PGP intensity decreased clinically significantly in the intervention group.

- In within-group analyses, we showed better sleep-duration improvement, fewer nightly wake-ups in the intervention group, deterioration of function and increased daytime sleepiness in the standard treatment group.

What do these results mean

This study provides valuable data outlining how the mattress and pillow may improve nightly posterior PGP in pregnant women. Considering the wide-ranging adverse effects of PGP in pregnant women, the findings, despite the obvious lack of statistical power, have definite clinical and practical values.

\section{Introduction}

About $30-40 \%$ of all women who are pregnant of all socioeconomic classes worldwide suffer from Pelvic girdle pain (PGP). ${ }^{1,2}$ It is also the number one cause of sick leave during pregnancy. ${ }^{3}$ According to the European guidelines for diagnosis and treatment of PGP, ${ }^{4}$ PGP usually debuts during or after pregnancy but can also debut as a result of trauma, arthritis or osteoarthritis. PGP is located between the posterior iliac crest and the fold of the seat, especially near the sacroiliac joints. It can spread down the back of the thigh and can also be experienced together with/or separately in the symphysis. The ability to stand, walk and sit is impaired. The diagnosis of PGP can be established when lumbar causes are eliminated and where specific clinical tests are used to reproduce the pain or dysfunction that occurs with PGP. ${ }^{4,5}$ PGP usually debuts during gestational weeks $12-24^{6}$ and typically worsens as the pregnancy proceeds. ${ }^{5}$ One in four women experience severe PGP and about 20-25\% require medical help. ${ }^{4}$

Previous PGP and low back pain and trauma to the back or pelvis, being multiparous, having a high body mass index (BMI), a strenuous and physically demanding work, and emotional distress increase the risk of developing PGP. ${ }^{4,7}$ However, age, hormonal contraceptive use, and the time interval since the last pregnancy are not risk factors for PGP. ${ }^{4}$ Women with PGP commonly report significant absenteeism from work, difficulties with sexual life, and increased nightly PGP when turning in bed, resulting in frequent awakenings that often lead to daytime tiredness and increased difficulties in performing normal daily activities. ${ }^{8,9}$ The sleep disturbances in turn induce physical symptoms. ${ }^{10,11}$, Some women also experience significant stress, worrying that the PGP is a signal of problems with their unborn baby, ${ }^{12}$ a state that may discourage them from future pregnancies. ${ }^{12}$

At present, there are no curative treatments for PGP during pregnancy, but a Cochrane review concluded that acupuncture or a combination of several interventions (manual therapy, exercise, and education) may be helpful. ${ }^{13}$ To our knowledge, there are no studies that have evaluated treatments for nightly PGP.

A mattress and a pillow consisting of viscoelastic (memory) and normal foam that shapes around the body, wherein the pillow is separated from the mattress to reduce the pressure on the shoulder when sleeping on the side, have been developed (Figure 1). It has been found to be efficient for position-dependent patients with snoring and sleep apnoea, ${ }^{14,15}$ since it is shaped to allow the user to sleep in the prone and side positions, which patients did for more than $95 \%$ of the night. ${ }^{14,15}$ One pregnant woman who had severe nightly pain due to PGP, tried to sleep on the present mattress and pillow, and she improved dramatically. This inspired us to perform the present study, although there exists, as far as we know, no earlier scientific evaluation of treating nightly PGP with a special mattress and pillow.

The aim of this randomised controlled crossover study was to assess the effectiveness of the memory-foam mattress and pillow plus standard treatment in comparison with solely standard treatment for nightly PGP during pregnancy.

\section{Materials and Methods}

A randomised controlled crossover study was planned at a university hospital in Sweden. The recruitment started in May 2018. However, due to the severe acute respiratory syndrome coronavirus 2 (SARS-CoV-2) 2019, participant recruitment had to be stopped on March 3, 2020, which meant that the sample size for adequate statistical power 


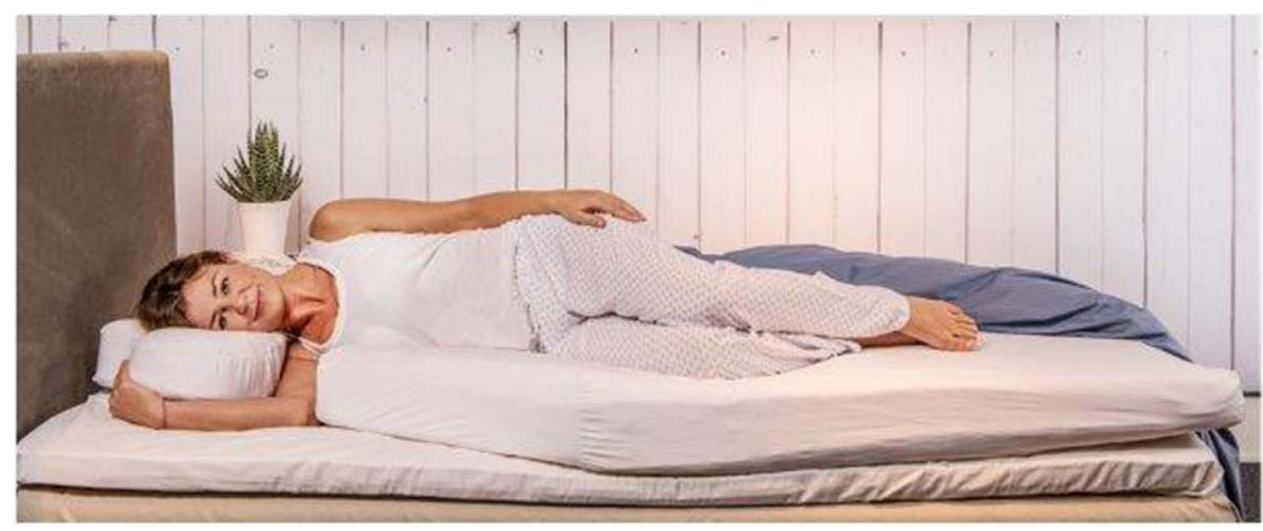

Figure I The mattress and pillow. Image courtesy from Dr Hasse Ejnell.

was not achieved. Due to the higher-than-expected dropout rate, we further decided not to continue the cross-over part of the trial. The study follows CONSORT guidelines ${ }^{16}$ and is registered at IRSCTN (No. ISRCTN13438272).

\section{Inclusion Criteria}

Inclusion criteria were healthy women with singleton pregnancies in gestational weeks 12 to 29 who experienced modest nightly PGP intensity, ie $\geq 40 \mathrm{~mm}$ on a 100 $\mathrm{mm}$ pain visual analogue scale (VAS) during 5-7 days before the screening visit. Women participating in the study had to understand and read Swedish and were diagnosed with PGP according to the European guidelines for diagnosis and treatment of PGP.

\section{Exclusion Criteria}

Women with systemic disorders, a history of orthopaedic disease, or surgery of the spine or pelvic girdle or other pain conditions, were excluded.

\section{Participants}

Midwives at the antenatal care units informed women with lumbopelvic pain of the intervention study. Interested women contacted the project coordinator $(\mathrm{CF})$ by telephone or e-mail and received further information about the study. Screening visits were booked for women who agreed to participate. A questionnaire for registration of demographics and patient-reported outcomes (PROMs) was sent to the women by post. Those willing to participate provided informed written consent in the postal questionnaire before completing it. The PROMs included BMI, parity, history of back and/or PGP, use of analgesics, lifestyle issues, sick-leave information, as well as healthrelated quality of life, function, pain catastrophic thinking, and daytime sleepiness assessed using reliable and valid instruments. The women also registered their nightly anterior and posterior pain intensity every morning and their anterior and posterior pain every evening, estimated sleep duration, and number of wake-ups during the night in a diary over 5-7 days before the screening visit. The women brought the postal questionnaire with them to the screening visit.

Women who were eligible and wanted to participate in the study were assessed by the project coordinator (CF), an independent specially trained physiotherapist. This assessment included a pain drawing and a standardised physical examination for the diagnosis of PGP as defined by the European guidelines. ${ }^{4}$ The examination included a modified Trendelenburg test and the MAT test ${ }^{17}$ to assess anterior pain and the Posterior Pelvic Pain Provocation test (PPPP-test) and Patrick's Faber test to assess posterior pain intensity. ${ }^{18,19}$ These pain provocation tests were used to discriminate posterior pain from low back pain, since they have shown sensitivity to provoking posterior pelvic structures. ${ }^{18}$ The Active Straight Leg Raising test, a functional test, was used to evaluate the participant's ability to raise her right and left legs separately $(0=$ "not difficult at all" to $5=$ "unable to perform"). ${ }^{20}$ These tests have been suggested by the European guidelines ${ }^{4}$ for use in clinical practice and as core outcomes for the evaluation of treatment effects for pregnancy-related PGP. ${ }^{21}$ They have also been used for classification of PGP in our earlier randomised controlled trials (RCTs) of treatment strategies for PGP during pregnancy. ${ }^{22-24}$

\section{Randomisation}

A statistician administered pre-coded numbered identical opaque envelopes to allocate participants to the 
intervention group or control group. A computer-generated sequence was used. The sequence was stratified to ensure balance in the frequency of sick leave between the groups. Sequences were derived from a table of correlatively ordered permutations of the letters A and B in groups of 10 , with each letter appearing five times. Women who fulfilled all inclusion criteria were allocated to standard treatment plus the mattress and pillow (intervention group, $\mathrm{n}=34$ ) for 4 weeks or to solely standard treatment (control group, $\mathrm{n}=32$ ) for 4 weeks directly after screening by the study coordinator $(\mathrm{CF})$.

\section{Interventions}

The standard treatment consisted of the same personcentred standard treatment used in previous studies. ${ }^{22-24}$ It included provision of standard information about PGP and the anatomy of the back and pelvis. In dialogue, the physiotherapist (CF) informed the women about the importance of rest and the relationships among impairment, load demand and actual loading capacity. The women were invited to describe their life situation and experiences regarding their pelvic discomfort. Advice was based on each women's priorities and activities in daily life. A home training program was offered to all women. The program included exercises for proper posture (finding the balance line), exercises to strengthen hip muscles and trunk (squats and activation of deep abdominal muscles standing on all fours), toe lifts, push-ups against wall, and stretching of hip benders and the back of thighs. Exercises and loads were adjusted to fit each woman. The women were also informed that they could contact CF if they wanted additional advice or crutches.

The mattress and pillow consisted of two or three layers of foam (Recticel, Sweden, C45190/V50080/ V55035). The upper thick layer is composed of a memory viscoelastic foam, which is temperaturesensitive and transforms the mattress to fit the body shape. The mattress and pillow were at a distance of approximately $10 \mathrm{~cm}$ apart in order to allow comfortable positioning of the arm and shoulder when sleeping in the side position. Both were offered in two models: soft (two layers) and medium-firm (three layers) and were placed upon the patient's normal bed (Figure 1). The women chose one model of mattress. The physical therapist instructed the women on using the mattress and pillow, and the women were able to test it. The women were instructed to sleep on the mattress for a minimum of $4 \mathrm{~h}$ / night during the following 4 weeks. They were called after
2 to 7 days to follow up on the mattress and pillow and give them advice about adjustments if needed. The women could borrow the mattress and pillow after the intervention period throughout the pregnancy. The women received a diary for registration of evening and nightly anterior and posterior pain during the fourth treatment week (5 to 7 days) together with a questionnaire for re-evaluation of the PROMs after four weeks of treatment. Questionnaires were either sent to CF by post or handed to CF upon return of the mattress and pillow.

\section{Outcome Measures}

The primary outcome measure was nightly posterior pain intensity, on a 100 -mm VAS (from $0=$ "no pain" to $100=$ "the worst possible pain"). Secondary outcomes were as follows: nightly anterior, evening anterior and evening posterior pain intensity (VAS, 0-100 mm), estimated sleep duration (minutes), and the number of nightly wake-ups. Symptoms and limitations in daily activities were measured with the Pelvic Girdle Questionnaire (PGQ) (0-75 points). ${ }^{25}$ The PGQ is a PGP specific instrument, which includes 20 activity items and 5 symptom items scored on a 4-point Likert scale. Each question is scored from "Not at all" (0) to "To a large extent" (3). The European Quality of Life measure-VAS is a vertical scale (from $0=$ "The poorest imaginable health state to $100=$ The optimal health state"). ${ }^{26}$ The Pain Catastrophizing Scale (PCS), which has 13 items, was used to assess the women's reports of pain catastrophic thinking relating to $\mathrm{PGP} .{ }^{27}$ Each item is rated on a 4-point Likert scale (from $0=$ "not at all" to $4=$ "constantly"). The total score (range, 0-65 points) was used in this study. Daytime sleepiness was rated on the Epworth Sleepiness Scale (ESS; 0-24 points). ${ }^{28}$ Each item was measured on a 3-point Likert scale (from $0=$ "no risk" to $3=$ "high risk").

\section{Statistical Analyses Power Calculation}

In order to detect a change of nightly posterior PGP intensity of $25 \mathrm{~mm}$ on VAS, $\alpha=0.05$ and $\beta=0.80$, the size of the study population will be 30 subjects per group. Considering a dropout rate of $20 \%$, we planned to include 72 women in the study. However, because of the restrictions imposed by the SARS-CoV-2 pandemic, ie, women with PGP were not allowed to visit the hospital, recruitment ended when 75 women were assessed for eligibility and 66 women were included. 
We defined the baseline mean VAS scores for nightly and evening anterior and posterior PGP intensity for each woman by calculating the mean of her pain scores recorded in the pain diary prior to the screening visit (57 days). The same calculation was performed for mean pain during the fourth week of treatment. The analysis was by the original assigned groups. The primary analysis was between-group comparisons for change from baseline to 4 weeks. We calculated the medians, 95\% confidence intervals, max; min, means, and standard deviation (SD) values when possible. We calculated the medians, $95 \%$ confidence intervals, max; min, means, and standard deviation (SD) values when possible. A chi-squared test or Fisher's exact test was used for categorical variables, and the Mann-Whitney $U$-test was used to compare differences between groups in continuous variables. The $t$-test was used to calculate $95 \%$ confidence intervals for mean differences between the groups. To enrich the data, a secondary analysis of within-group differences in both groups from baseline to 4 weeks was included, which was performed with Sign test for dichotomous variables and the Wilcoxon's signed rank test for continuous variables. All tests were two-sided using a significance level of 5\%. Statistical analysis was performed by use of SPSS, version 26.0 (SPSS Inc., Chicago, IL, USA).

\section{Results}

A total of 75 women were assessed for inclusion; whereof 66 met the inclusion criteria and were included in the trial. Figure 2 shows the participants progress through trial and withdrawals. Seven of the $32(22 \%)$ women in the control group discontinued the intervention. Of these, two women stated that they no longer experienced nightly PGP, while the other five women did not give a reason for declining participation. In contrast, 12 of the 34 women in the intervention group (35\%) discontinued the intervention, one woman due to premature contractions before starting to sleep with the mattress and pillow and 11 women because they found the mattress and pillow uncomfortable or because it resulted in increased PGP or neck and/or

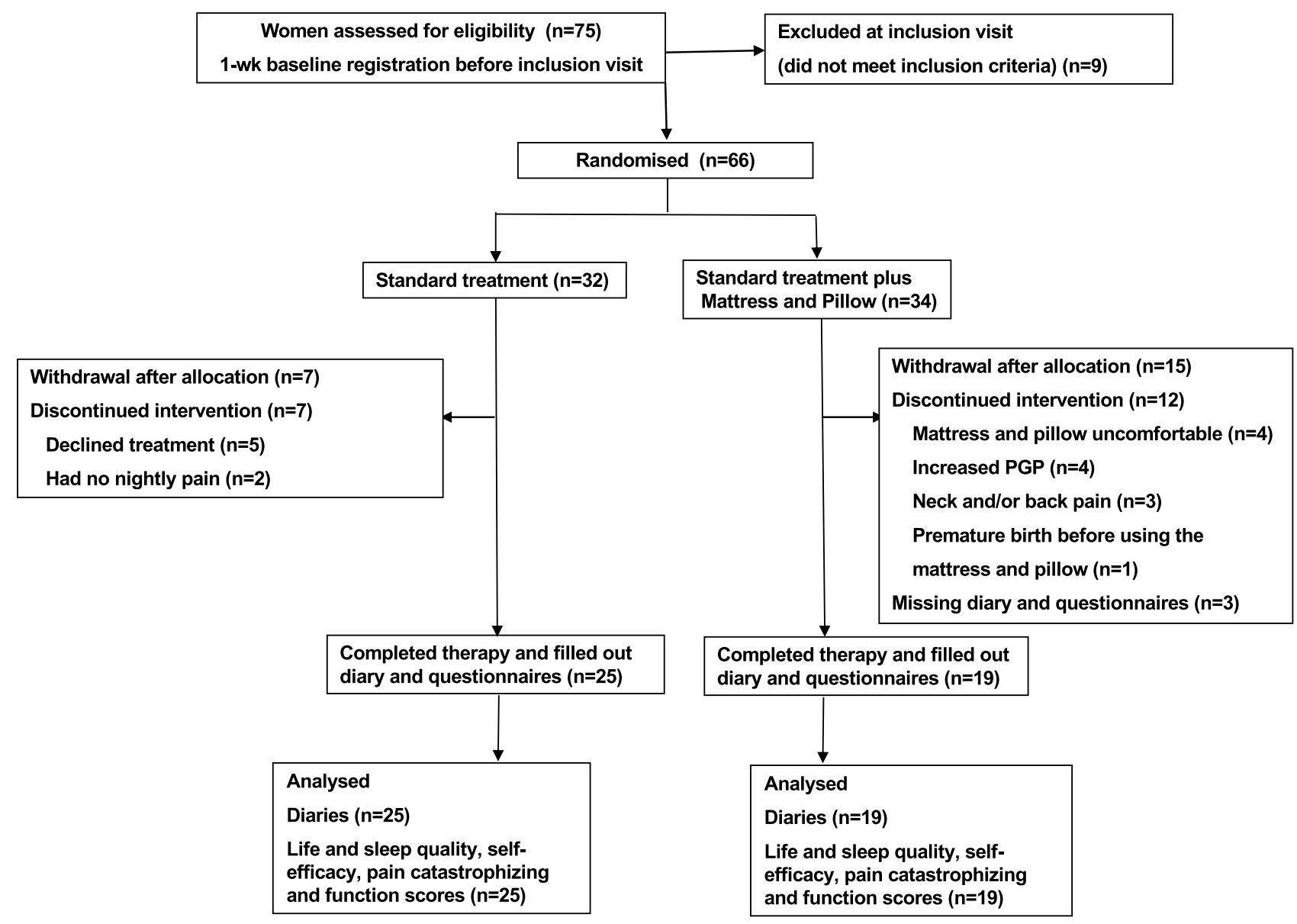

Figure 2 Participants' progress through trial and withdrawals. 
back pain. Baseline characteristics (Table 1) were comparable in the treatment groups. In addition, the women who completed four weeks of treatment and those who dropped out showed no differences in baseline characteristics (data not shown).

Table 2 shows the results between the groups in change from baseline to 4 weeks. For the primary outcome, ie, nightly posterior pain intensity, the intervention group showed a significant decrease compared to the control group ( $p=0.028$ ). For secondary outcomes sleep duration increased significantly within both groups $(p=0.022$ and $\mathrm{p}=0.014$ ) although the increase was significantly larger in the intervention group compared to the control group $(\mathrm{p}=$ 0.046). No other significant between-group differences were seen in the secondary outcomes. The results of the within-group change from baseline to 4 weeks of treatment showed that pain catastrophic thinking decreased in both groups $(\mathrm{p}=0.005$ and $\mathrm{p}=0.010)$. In the intervention group, evening posterior pain intensity decreased significantly $(p=0.008)$ as well as the estimated number of nightly wake-ups $(\mathrm{p}=0.049)$. The control group showed a significant deterioration in function $(p=0.018)$ and an

Table I Baseline Characteristics of the Study Groups

\begin{tabular}{|c|c|c|}
\hline Variables & $\begin{array}{l}\text { Intervention Group } \\
\mathrm{n}=\mathbf{3 4}\end{array}$ & $\begin{array}{l}\text { Control Group } \\
\mathrm{n}=32\end{array}$ \\
\hline Maternal age (years) & $31.4(3.9)$ & $30.8(4.8)$ \\
\hline \multicolumn{3}{|l|}{ Education } \\
\hline Primary School & $3(9)$ & $3(9)$ \\
\hline High School & $8(23)$ & $6(19)$ \\
\hline University & $18(53)$ & $16(50)$ \\
\hline Other & $5(15)$ & $7(22)$ \\
\hline Nulliparous women & $13(38)$ & $12(38)$ \\
\hline Gestational age (week) & $22.6(5.5)$ & $21.9(5.2)$ \\
\hline BMI at inclusion & $27.1(3.7)$ & $26.2(4.3)$ \\
\hline Use of analgesics* & $9(26)$ & $6(19)$ \\
\hline \multicolumn{3}{|c|}{ Pain provocation tests for anterior pain } \\
\hline Modified Trendelenburg test & $24(7 \mathrm{I})$ & $20(63)$ \\
\hline MAT-test & $24(71)$ & $26(8 I)$ \\
\hline \multicolumn{3}{|c|}{ Pain provocation tests for posterior pain } \\
\hline Modified Trendelenburg test & $27(79)$ & $24(75)$ \\
\hline PPPP-test & $33(97)$ & $28(88)$ \\
\hline Patrick's Faber test & $28(82)$ & $27(84)$ \\
\hline \multicolumn{3}{|l|}{ Functional test } \\
\hline ASLR- test $(0-10)$ & $4.9(3.1)$ & $4.3(3.2)$ \\
\hline \multicolumn{3}{|l|}{ Primary outcomes } \\
\hline Nightly anterior pain, VAS, $0-100 \mathrm{~mm}$ & $44.3(26.1)$ & $46.5(25.4)$ \\
\hline Nightly posterior pain, VAS & $61.2(16.9)$ & $60.4(21.5)$ \\
\hline \multicolumn{3}{|l|}{ Secondary outcomes } \\
\hline Evening anterior pain, VAS & $45.9(27.3)$ & $51.2(26.9)$ \\
\hline Evening posterior pain, VAS & $60.9(17.6)$ & $65.0(20.9)$ \\
\hline ESS & $12.0[7 ; 16]$ & $10.5[8 ; 13]$ \\
\hline EQ-VAS & $50.0[30 ; 60]$ & $46.5[34 ; 65]$ \\
\hline Nightly wake ups & $3.9(2.1)$ & $3.2(1.6)$ \\
\hline PCS, total score & $24.5[17 ; 34]$ & $28.0[19 ; 36]$ \\
\hline PGQ & $52.5[47 ; 58]$ & $49.0[42 ; 57]$ \\
\hline Sleep duration, minutes & $360(85.2)$ & $359(78.0)$ \\
\hline Women on sick leave & $16(47 \%)$ & $15(47)$ \\
\hline
\end{tabular}

Notes: Values are given as n (\%), mean (SD) or median [25 to 75 quartiles] when appropriate. All p-values not significant. *Paracetamol. Intervention group, Standard treatment plus mattress and pillow, Control group, Standard treatment.

Abbreviations: ASLR-test, active straight leg test; BMI, body mass index; ESS, Epworth sleepiness scale; EQ-VAS, euroqol visual analogue scale; PGQ, pelvic girdle questionnaire; PCS, pain catastrophizing scale; PPPP-test, posterior pelvic pain provocation test; VAS, visual analogue scale: 0 to $100 \mathrm{~mm}$. 


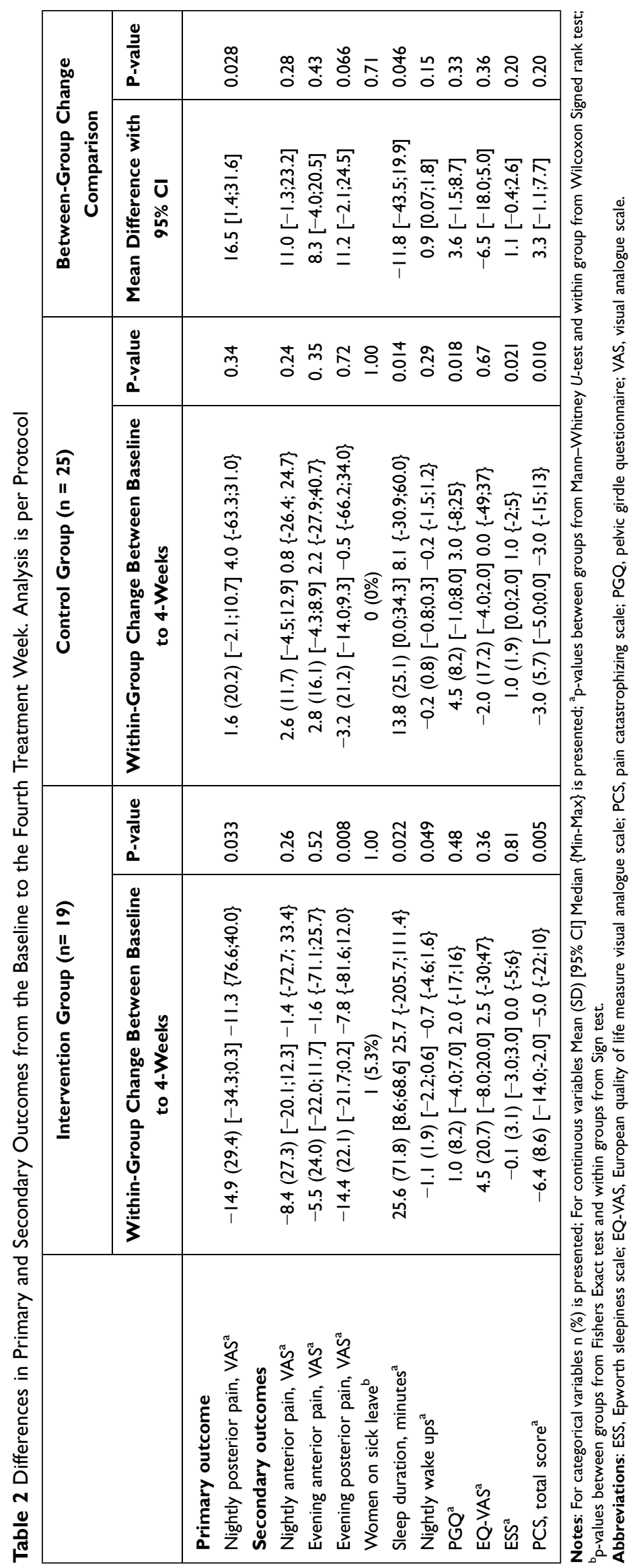


increase in daytime sleepiness $(\mathrm{p}=0.021)$ from baseline to 4 weeks.

\section{Adverse Effects}

Twelve minor adverse events were reported in the intervention group: pain in the neck, back, and shoulder regions $(n=4)$, deteriorated sleep $(n=4)$, and temporarily increased PGP intensity $(n=4)$. No adverse events were reported in the control group.

\section{Discussion}

The primary finding of this study was a clinically important lower nightly posterior pain intensity among women receiving the mattress and pillow plus standard treatment in comparison with solely standard treatment. Moreover, the reduction of evening and nightly posterior pain in the intervention group were clinically significant as $>30 \%$ or $13 \mathrm{~mm}$ on a VAS represents, on average, the minimum clinically relevant change in acute pain. ${ }^{29}$ Within-group analysis showed favourable results regarding mean posterior pain intensity, sleep duration (minutes), number of wake-ups, and pain catastrophic thinking in the intervention group. This finding may be of importance for women since pain is expected to aggravate and cause deterioration of sleep as the pregnancy proceeds. ${ }^{10,11}$ However, no effects regarding nightly anterior pain, health-related quality of life, function, pain catastrophic thinking, or sleep disturbances were seen between groups.

Many women avoid sleeping in the supine position during the last months of pregnancy and often experience difficulties in finding a comfortable position in the bed. ${ }^{8}$ Pregnant women with PGP also experience pain and limitations in sleep positioning. An advantage of this mattress and pillow was that it was developed to avoid sleeping in a supine position. One reason for drop-outs due to uncomfortable mattress and pillow could be that the present mattress and pillow were not designed to fit pregnant women and were developed to improve sleep in patients with positional sleep apnoea who were supposed to sleep as little as possible in supine position. A lot of patients with sleep apnoea have weights over $100 \mathrm{~kg}$. In order to fit these patients, the mattress was designed to be $15 \mathrm{~cm}$ in height. However, we stipulated that the mattress should fit most women in the study since PGP is more frequent in pregnant women with increased BMI. ${ }^{30}$ The viscoelastic foam or memory foam followed the body and supported the pelvis and abdomen in pregnant women. However, acclimatisation to a new mattress and pillow normally takes up to one month for a non-pregnant person.

\section{Strengths and Limitations}

The primary strengths of the study are the randomised controlled design, the use of the same individual standard treatment and PROMs (eg the PGQ, ${ }^{25}$ EQ-VAS, ${ }^{26}$ and the PCS) that have been used in earlier RCTs for PGP and have been recommended by the Swedish quality registry for pain rehabilitation and the European guidelines for PGP. ${ }^{4}$ They are also recommended as core outcomes for PGP in a newly published review. ${ }^{21}$ In addition, a systematic review reports on 21 studies that used the VAS for measurements of lumbopelvic pain intensity. ${ }^{31}$ To enrich the data, we performed within-group analyses after 4 weeks of treatment or control period. The results showed increased sleep duration and fewer nightly wake-ups in contrast to the decreased function and increased daytime sleepiness in the control group. Another strength of the study is that it was not only designed to investigate painrelieving effects, function, health-related quality of life, and pain catastrophic thinking, which have been measured in earlier studies, ${ }^{13}$ but also to evaluate sleep duration, nightly wake-ups, and daytime tiredness. Favourable results for the latter could have enhanced the direct clinical relevance since low sleep quality and sleep disturbances can provoke and worsen physical symptoms. ${ }^{10,11}$ Nevertheless, despite using the same classification and outcome measures as those in earlier studies, ${ }^{22-24}$ the differences in inclusion criteria precluded comparison of the PROMs from this study with those in the earlier studies. Moreover, the significant p-values could have occurred accidentally since several different variables and time points were analysed independently. However, the pain-relieving effect appears to be consistent across the results of between-group and within-group analyses. Yet, there are several limitations in this study. The sample size for adequate statistical power was not achieved due to the restrictions imposed by the SARS-CoV-2 pandemic, and the drop-out rate was high. The data was therefore analysed per protocol to avoid a misinterpretation. Future trials, with larger groups, are needed to be able to analyse the full effect of the intervention.

\section{Conclusions}

Adjunctive use of the mattress and pillow may decrease nightly posterior PGP. However, the drop-out rate was high, and the findings should be interpreted with caution. 
In conclusion, significantly lower nightly posterior pain intensity was noted after the use of a viscoelastic (memory) and normal foam mattress and pillow as an adjunct to standard treatment. Nightly PGP can have adverse effects on various aspects of the health and quality of life of pregnant women, and although the results of this study should be interpreted with caution considering the high drop-out rate and the inadequate statistical power, the findings indicate the potential for the use of such interventions to improve PGP in pregnant women.

\section{Abbreviations}

BMI, body mass index; CPAP, continuous positive airway pressure; ESS, Epworth sleepiness scale; EQ-VAS, European quality of life measure visual analogue scale; PCS, pain catastrophizing scale; PGP, pelvic girdle pain; PGQ, pelvic girdle questionnaire; PPPP-test, posterior pelvic pain provocation test; PROMS, patient reported outcomes; RCT, randomised controlled trial; VAS, visual analogue scale; SD, standard deviation.

\section{Data Sharing Statement}

Data can be made available on reasonable request to the corresponding author.

\section{Ethics Approval and Informed Consent}

Approval for this study was obtained from the Regional Medical Ethics Committee in Gothenburg, Sweden (0915152009/099-09). The study follows CONSORT guidelines and is registered at IRSCTN (No. ISRCTN13438272). All participants were informed as to the purpose of the study, in accordance with the Declaration of Helsinki and gave their written consent.

\section{Acknowledgments}

We thank the medical doctors and midwives at the antenatal units for their invaluable help with the recruitment of the participants. We also express our appreciation to all the women for their contributions to this study. We acknowledge statistician Nils-Gunnar Pehrsson at Statistiska Konsultgruppen for assistance with the study design, power calculation and randomisation. Figure 1 image is owned by and with courtesy from Dr Ejnell.

\section{Author Contributions}

All authors made substantial contributions to conception and design, acquisition of data, or analysis and interpretation of data; took part in drafting the article or revising it critically for important intellectual content; agreed to submit to the current journal; gave final approval for the version to be published; and agreed to be accountable for all aspects of the work.

\section{Funding}

This research was supported by the Foundation of the Health and Medical care committee of the Region of Västra Götaland, Sweden (VGFOUREG757191, VGFOUREG-846141).

\section{Disclosure}

Dr Feldthusen, Dr Fagevik-Olsen and Dr Elden declare that they have no competing interest. Dr Ejnell is the owner of the mattress and pillow for treatment of sleep apnoea. The authors report no other conflicts of interest in this work.

\section{References}

1. Bjorklund $\mathrm{K}$, Bergstrom S. Is pelvic pain in pregnancy a welfare complaint? Acta Obstet Gynecol Scand. 2000;79(1):24-30. doi:10.1080/j.1600-0412.2000.079001024.x

2. Gashaw M, Gedlu S, Janakiraman B. Burden of pelvic girdle pain during pregnancy among women attending ante-natal clinic, Ethiopia: across-sectional study. BMC Pregnancy Childbirth. 2020;20(1):494. doi:10.1186/s12884-020-03184-4

3. Malmqvist S, Kjaermann I, Andersen K, Okland I, Larsen JP, Bronnick K. The association between pelvic girdle pain and sick leave during pregnancy; a retrospective study of a Norwegian population. BMC Pregnancy Childbirth. 2015;15:237. doi:10.1186/ s12884-015-0667-0

4. Vleeming A, Albert HB, Ostgaard HC, Sturesson B, Stuge B. European guidelines for the diagnosis and treatment of pelvic girdle pain. Eur Spine J. 2008;17(6):794-819. doi:10.1007/s00586-0080602-4

5. Sihvonen T, Huttunen M, Makkonen M, Airaksinen O. Functional changes in back muscle activity correlate with pain intensity and prediction of low back pain during pregnancy. Arch Phys Med Rehabil. 1998;79(10):1210-1212. doi:10.1016/S0003-9993(98) 90264-7

6. Robinson H, Mengshoel A, Veierod M, Vollestad N. Pelvic girdle pain: potential risk factors in pregnancy in relation to disability and pain intensity three months postpartum. Man Ther. 2010;15(6):522-528. doi:10.1016/j.math.2010.05.007

7. Bjelland E, Eskild A, Johansen R, Eberhard-Gran M. Pelvic girdle pain in pregnancy: the impact of parity. Am $J$ Obstet Gynecol. 2010;203(2):146e1-e6. doi:10.1016/j.ajog.2010.03.040

8. Elden H, Lundgren I, Robertson E. Life's pregnant pause of pain: pregnant women's experiences of pelvic girdle pain related to daily life: a Swedish interview study. Sex Reprod Healthc. 2013;4(1):29-34. doi:10.1016/j.srhc.2012.11.003 
9. Varley M, Hunter L. How does pelvic girdle pain impact on a woman's experience of her pregnancy and the puerperium? EvidBased Midwifery. 2019;17(2):60.

10. Kamysheva E, Skouteris H, Wertheim E, Paxton S, Milgrom J. Examination of a multi-factorial model of body-related experiences during pregnancy: the relationships among physical symptoms, sleep quality, depression, self-esteem, and negative body attitudes. Body Image. 2007;5(2):152-163. doi:10.1016/j.bodyim.2007.12.005

11. Roth T. Insomnia: definition, prevalence, etiology, and consequences. J Clin Sleep Med. 2007;3(5 Suppl):S7-S10. doi:10.5664/jcsm.26929

12. Svensson H, Andersson G, Hagstad A, Jansson P. The relationship of low-back pain to pregnancy and gynecologic factors. Spine. 1990;15 (5):371-375. doi:10.1097/00007632-199005000-00006

13. Pennick V, Liddle SD. Interventions for preventing and treating pelvic and back pain in pregnancy. Cochrane Database Syst Rev. 2013;8:CD001139. doi:10.1002/14651858.CD001139.pub3

14. Bidarian-Moniri A, Nilsson M, Attia J, Ejnell H. Mattress and pillow for prone positioning for treatment of obstructive sleep apnoea. Acta Otolaryngol. 2015;135(3):271-276. doi:10.3109/00016489.2014.968674

15. Bidarian-Moniri A, Nilsson M, Rasmusson L, Attia J, Ejnell H. The effect of the prone sleeping position on obstructive sleep apnoea. Acta Otolaryngol. 2015;135(1):79-84. doi:10.3109/00016489.2014.962183

16. Moher D, Schulz KF, Altman D, Group C. The CONSORT statement: revised recommendations for improving the quality of reports of parallel-group randomized trials. JAMA. 2001;285(15):1987-1991. doi:10.1001/jama.285.15.1987

17. Fagevik Olsen M, Gutke A, Elden H, et al. Self-administered tests as a screening procedure for pregnancy-related pelvic girdle pain. Eur Spine J. 2009;18(8):1121-1129. doi:10.1007/s00586-009-0948-2

18. Albert H, Godskesen M, Westergaard J. Evaluation of clinical tests used in classification procedures in pregnancy-related pelvic joint pain. Eur Spine J. 2000;9(2):161-166. doi:10.1007/ s005860050228

19. Ostgaard HC, Zetherstrom G, Roos-Hansson E. The posterior pelvic pain provocation test in pregnant women. Eur Spine J. 1994;3 (5):258-260. doi:10.1007/BF02226575

20. Mens JM, Vleeming A, Snijders C, Koes BW, Stam HJ. Reliability and validity of the active straight leg raise test in posterior pelvic pain since pregnancy. Spine. 2001;26(10):1167-1171. doi:10.1097/ 00007632-200105150-00015

21. Remus A, Smith V, Gutke A, et al. A core outcome set for research and clinical practice in women with pelvic girdle pain: PGP-COS. PLoS One. 2021;16(2):E0247466. doi:10.1371/journal. pone. 0247466
22. Elden H, Ladfors L, Olsen MF, Ostgaard HC, Hagberg H. Effects of acupuncture and stabilising exercises as adjunct to standard treatment in pregnant women with pelvic girdle pain: randomised single blind controlled trial. BMJ. 2005;330(7494):761. doi:10.1136/ bmj.38397.507014.E0

23. Elden H, Fagevik-Olsen M, Ostgaard HC, Stener-Victorin E, Hagberg H. Acupuncture as an adjunct to standard treatment for pelvic girdle pain in pregnant women: randomised double-blinded controlled trial comparing acupuncture with non-penetrating sham acupuncture. BJOG. 2008;115(13):1655-1668. doi:10.1111/j.14710528.2008.01904.x

24. Elden H, Ostgaard HC, Glantz A, Marciniak P, Linner AC, Olsen MF. Effects of craniosacral therapy as adjunct to standard treatment for pelvic girdle pain in pregnant women: a multicenter, single blind, randomized controlled trial. Acta Obstet Gynecol Scand. 2013;92(7):775-782. doi:10.1111/aogs.12096

25. Gutke A, Stuge B, Elden H, Sandell C, Asplin G, Fagevik olsen M. The Swedish version of the pelvic girdle questionnaire, cross-cultural adaptation and validation. Disabil Rehabil. 2020;42(7):1464-5165. doi:10.1080/09638288.2018.1515991

26. Rabin R, De Charro F. EQ-5D: a measure of health status from the EuroQol group. Ann Med. 2001;33(5):337-343. doi:10.3109/ 07853890109002087

27. Sullivan MJL, Bishop SR, Pivik J. The pain catastrophizing scale. Psychol Assess. 1995;7(4):524-532. doi:10.1037/10403590.7.4.524

28. Johns M. A new method for measuring daytime sleepiness: the Epworth sleepiness scale. Sleep. 1991;14(6):540. doi:10.1093/sleep/ 14.6 .540

29. Gallagher E, Liebman M, Bijur PE. Prospective validation of clinically important changes in pain severity measured on a visual analog scale. Ann Emerg Med. 2001;38(6):633-638. doi:10.1067/ mem.2001.118863

30. Biering K, Nohr E, Olsen J, Andersen A, Hjollund N, Juhl M. Pregnancy-related pelvic pain is more frequent in women with increased body mass index. Acta Obstet Gynecol Scand. 2011;90 (10):1132-1139. doi:10.1111/j.1600-0412.2011.01141.x

31. Wuytack F, O’Donovan M. Outcomes and outcomes measurements used in intervention studies of pelvic girdle pain and lumbopelvic pain: a systematic review. Chiropr Man Therap. 2019;27(1):62. doi:10.1186/s12998-019-0279-2

\section{Publish your work in this journal}

The International Journal of Women's Health is an international, peerreviewed open-access journal publishing original research, reports, editorials, reviews and commentaries on all aspects of women's healthcare including gynecology, obstetrics, and breast cancer. The manuscript management system is completely online and includes a very quick and fair peer-review system, which is all easy to use. Visit http://www.dovepress.com/testimonials.php to read real quotes from published authors. 